\title{
Effects of Cyclopentolate Hydrochloride Dosage on Anterior Segment Parameters in Young Adults (Measured with Pentacam)
}

This article was published in the following Dove Press journal: Clinical Ophthalmology

\author{
Waleed M Alghamdi \\ Saif H Alrasheed \\ Vishakh Nair \\ Muhammed S Alluwimi \\ Department of Optometry, College of \\ Applied Medical Sciences, Qassim \\ University, Buraydah, Saudi Arabia
}

Correspondence: Saif H Alrasheed

Qassim Unversity, P.O. Box II62,

Buraydah, Saudi Arabia

Tel +966505201475

Fax +966-16-3800050

Email s.rasheed@qu.edu.sa
Purpose: To assess the effects of $0.5 \%$ and $1 \%$ cyclopentolate on the main parameters of the anterior segment (central corneal thickness (CCT), anterior chamber angle (ACA), depth (ACD) and volume (ACV)) in low/moderate myopia and hyperopia along with the effect on IOP.

Patients and Methods: Both eyes of 30 subjects (15 myopic and 15 hyperopic) with mean age \pm standard deviation of $21.4 \pm 3.6$ years were enrolled. Each participant was administered two drops of cyclopentolate $1 \%$ in the right eye and two drops of cyclopentolate $0.5 \%$ in the left eye, 15 minutes apart. All participants underwent intraocular pressure (IOP) measurement using noncontact tonometry, and anterior chamber parameter measurement using Pentacam.

Results: Following the use of $0.5 \%$ and $1 \%$ cyclopentolate among the hyperopic group, there was a statistically significant increase in ACD for $1 \%$ (pre $2.762 \pm 0.28 \mathrm{~mm}$ and post 2.89 $\pm 0.25 \mathrm{~mm}$ ) and $0.5 \%$ (pre $2.71 \pm 0.28$ and post $2.86 \pm 0.27 \mathrm{~mm}$ ) and ACV for $1 \%$ (pre 141.40 $\pm 20.59 \mathrm{~mm}^{3}$ and post $154.35 \pm 19.69 \mathrm{~mm}^{3}$ ) and $0.5 \%$ (pre $137.40 \pm 20.48 \mathrm{~mm}^{3}$ and post 152.93 $\pm 20.50 \mathrm{~mm}^{3}$ ). In contrast, ACA decreased with both doses $1 \%$ and $0.5 \%$, but was not statistically significant ( $p$ for both $>0.05 \%$ ). With $0.5 \%$ and $1 \%$ cyclopentolate among the myopia group, there was a significant increase in ACD following cyclopentolate $1 \%$ (pre 3.18 $\pm 0.22 \mathrm{~mm}$ and post $3.25 \pm 0.21 \mathrm{~mm}$ ) and $0.5 \%$ (pre $3.200 \pm 0.22 \mathrm{~mm}$ and post $3.26 \pm 0.05 \mathrm{~mm}$ ), $p<0.05$. The ACV was significantly increased following $1 \%$ cyclopentolate, $p<0.001$. The ACA showed a statistically significant decrease following cyclopentolate $1 \%, P=0.01$, but not a significant decrease after cyclopentolate $0.5 \%, P=0.170$. There was a significant increase in the IOP after $1 \%, p<0.001$, while a decrease with $0.5 \%, p=0.008$.

Conclusion: A topical dosage of cyclopentolate $1 \%$ showed significant changes in ACA and ACV among the hyperopia and myopic groups compared to $0.5 \%$. Therefore, it is important to consider the use of a $0.5 \%$ cyclopentolate dosage to minimize changes to anterior chamber parameters.

Keywords: cycloplegic, anterior chamber angle, anterior chamber volume depth, central corneal thickness

\section{Introduction}

Cycloplegic agents have become essential diagnostic and therapeutic components for ocular examination. The uses of cycloplegic agents include, but are not limited to, fundus examination, anterior segment assessment, refraction, and diagnosis and treatment of amblyopia using pharmacological penalization. ${ }^{1,2}$ Evaluation of anterior segment structures has become crucial for the planning of cataract and refractive surgery, diagnosing and treating glaucoma and assessing corneal health. ${ }^{3-5}$ Full assessment of the anterior segment is important for diagnosing and monitoring 
patients with glaucoma, performing corneal refractive surgery, intraocular lens (IOL) calculation, and the lens position in cataract surgery. ${ }^{2,6-8}$ Cyclopentolate is commonly used as a cycloplegic agent for the examination of children, providing a one-hour cycloplegia similar to that from three days of atropine. ${ }^{9}$ Because cyclopentolate has relatively short durations of action compared to other cycloplegic agents such as atropine, it is widely used in clinical practice. $^{2,10,11}$

The most commonly used concentrations of cyclopentolate are $1 \%$ and $0.5 \%$. Several studies demonstrated that $1 \%$ cyclopentolate may cause an increase in the anterior chamber depth (ACD) and anterior chamber volume $(\mathrm{ACV})$, but a decrease in the anterior chamber angle (ACA). ${ }^{2,6,12-14}$ These changes pose a risk to the patient's eye, as the assessment of anterior segment structure has become critical for planning for refractive surgery, cataract surgery, diagnosing and treating glaucoma and assessing corneal health. ${ }^{3,4,15}$ Meanwhile, use of the $1 \%$ and $0.5 \%$ cyclopentolate concentrations showed no significant differences regarding the residual accommodation. ${ }^{16}$ However, to the best of our knowledge, there is no clinical guideline that specifies when to use $0.5 \%$ or $1.0 \%$ cyclopentolate for determining changes in the anterior chamber parameters. The differences in effect between the use of $1 \%$ cyclopentolate as compared to $0.5 \%$ cyclopentolate on anterior segment parameters have not previously been evaluated.

Because effects could be observed on the anterior segment parameters after the administration of the cyclopentolate agents, changes to the intraocular pressure (IOP) measurements are expected. Although cyclopentolate agents do not cause as much pupil dilation as other mydriatic agents do, several studies indicated that the use of $1 \%$ cyclopentolate agents might lead to little increase in the IOP. ${ }^{17,18}$ However, the effect of $0.5 \%$ cyclopentolate has not been addressed. It is not clear whether or not there would be an increase in the IOP measurements when $0.5 \%$ cyclopentolate is administrated. The knowledge regarding this concern is warranted to build a framework for clinicians who use cyclopentolate agents in order to perform convenient diagnostic examination.

This study aimed to assess differences in changes in the anterior chamber parameters when using $1 \%$ and $0.5 \%$ cyclopentolate hydrochloride instillation. These parameters include ACD, ACV and ACA width. In addition, we will assess the changes with these concentrations on central corneal thickness (CCT), pupil size and intraocular pressure (IOP) among hyperopic and myopic participants.

\section{Patients and Methods}

\section{Study Design}

This was a cross-sectional study, conducted among staff members and college students in Qassim University. The study included 60 eyes of 15 hyperopic and 15 myopic males aged 18-25 years, mean and standard deviation of $21.4 \pm 3.6$ years.

\section{Inclusion Criteria}

Subjects aged 18-25 years, hypermetropic from +0.50 to +3.50 DS and myopic from -0.50 to -3.50 DS with astigmatism no more than -0.75 D. Participants had to have $18 \mathrm{mmHg}$ or less for the IOP measurements.

\section{Exclusion Criteria}

Participants with ocular disease such as corneal pathology, glaucoma, uveitis and posterior segment pathology were excluded. Another exclusion criterion was previous eye surgery or eye trauma, and posterior segment pathology was excluded. Also, participants who were currently under topical or systemic medications, those with systemic diseases and those using contact lens for more than one year.

\section{Ethical Considerations}

Ethical permission for the performance of the study was obtained from the biomedical Ethics Committee at Qassim University (ref: 19-07-04). The study was conducted according to the Declaration of Helsinki guidelines. The goals and study protocol were explained to the participants and informed consent was obtained from all the participants, to facilitate a better understanding of the procedures and conditions of involvement in the study. The participants are participating in a voluntary way. They can withdraw from the study at any time without giving any reason. All forms and data sheets were shredded as soon as they were entered into the database system for analysis.

\section{Data Collection Procedures}

During data gathering processes, the subjects were requested to sign informed consents. Then, participants underwent a refractive error assessment using the Topcon KR8900 autorefractometer (Topcon Co., Tokyo, Japan), and an average of three IOP measurements which were taken using Pulsair Intellipuff (Keeler-Malvern, UK) noncontact tonometry. Anterior chamber parameter measurement included anterior chamber depth (ACD), anterior chamber volume (ACV), anterior chamber angle (ACA) 
width, central corneal thickness (CCT), front and back keratometry readings and pupil diameter using Pentacam (Oculus, Wetzlar, Germany), and the average of the three measurements was used as the final value for all parameters. Each participant was administered two drops of cyclopentolate $1 \%$ in the right eye and two drops of cyclopentolate $0.5 \%$ (Cyclogel; Alcon Laboratories, Fort Worth, TX, USA) in the left eye separated by 15 minutes apart, and after 30 minutes the above-mentioned measurements were repeated. All measurements were taken between $10 \mathrm{AM}$ and $2 \mathrm{PM}$ in identical lighting conditions. Malvern, UK

\section{Data Analysis}

Statistical analysis was performed with SPSS for Windows Version 21.0 (SPPS Inc., Chicago, IL, USA). All data were reported as averages \pm standard deviations (SD). A paired $t$-test was used to compare variables between the pre- and post-cycloplegia conditions. A value of $p<0.05$ was considered statistically significant.

\section{Results}

\section{Demographic Characteristics of Participants}

The study included 60 eyes of 15 hyperopic and 15 myopic subjects, all of them male, and their age ranged from 18 to 25 years with a mean \pm SD of $21.4 \pm 3.6$ years. The difference between the mean ages of hyperopic and myopic subjects was not statistically significant $(p=0.84)$.

\section{The Effects of Cyclopentolate $0.5 \%$ and I\% Dosage on Anterior Segment}

\section{Parameters Among the Hyperopic Group}

The mean ACA measurement for the hyperopic group decreased following cyclopentolate in both dosages but the difference was not statistically significant for either cyclopentolate $1 \%(p=0.156)$ or cyclopentolate $0.5 \%$ $(p=0.378)$ as shown in Table 1 and Figures 1 and 2. The mean ACD measurement increased following cyclopentolate $1 \%$ (pre $2.762 \pm 0.28 \mathrm{~mm}$ and post $2.89 \pm 0.25 \mathrm{~mm}$ ) and the difference was statistically significant, $P<0.001$ (Figure 3). On the other hand, the mean ACD measurement increased after cyclopentolate $0.5 \%$ (pre $2.71 \pm 0.28$ $\mathrm{mm}$ and post $2.86 \pm 0.27 \mathrm{~mm}$ ) and the mean difference was statistically significant, $P<0.001$ (Figure 4).

The mean ACV measurement was significantly increased after using both dosages of cyclopentolate,
$P<0.001$, as shown in Figures 5 and 6. The mean CCT measurement was increased following cyclopentolate $1 \%$ (pre $568.93 \pm 31.15 \mu$ and post $571.13 \pm 30.79 \mu$ ), the mean difference of which was statistically not significant, $P=0.096$. However, the mean CCT measurement remained the same before and after using cyclopentolate $0.5 \%$, the mean difference of which was statistically not significant, $P=0.864$.

The mean IOP measurement of the hyperopic group was significantly increased following cyclopentolate 1\% (pre $13.25 \pm 1.77 \mathrm{mmHg}$ and post $15.13 \pm 2.49 \mathrm{mmHg}$ ), which was statistically significant, $P<0.005$. However, the mean IOP measurement increased after cyclopentolate $0.5 \%$ (pre $14.05 \pm 2.03 \mathrm{mmHg}$ and post $14.74 \pm 1.84 \mathrm{mmHg}$ ), which was statistically not significant, $P=0.05$. With regard to the mean change of refractive error, both dosages of cyclopentolate significantly increased in hyperopia which was statistically significant ( $1 \%$ cyclopentolate $P<0.001$ and $0.5 \%$ cyclopentolate $p=0.002$ ). The mean pupil size measurement was significantly increased following both cyclopentolate dosages, which was statistically significant, $P<0.001$, as shown in Table 1.

\section{The Effects of Cyclopentolate $0.5 \%$ and 1\% Dosage on Anterior Segment}

\section{Parameters Among the Myopic Group}

The mean ACA measurement for the myopic group decreased following cyclopentolate $1 \%$ (pre $40.82 \pm 1.90^{\circ}$ and post $38.80 \pm 2.65^{\circ}$ ), and the mean difference was statistically significant. $P=0.001$ (Figure 1). However, the mean of ACA measurement following $0.5 \%$ cyclopentolate decreased (pre $39.78 \pm 2.08^{\circ}$ and post $38.25 \pm 3.60^{\circ}$ ), and the mean difference was not statistically significant, $P=0.170$ (Figure 2). For the mean ACD measurement, there was a significant increase following cyclopentolate in both dosages, and the mean difference was statistically significant in both $1 \%$ cyclopentolate $(p=0.001)$ and $0.5 \%$ cyclopentolate $(p=0.003)$ as shown in Table 2 .

The mean ACV measurement of the myopic group showed a significant increment $(P<0.01)$ following $1 \%$ cyclopentolate (pre $201.60 \pm 23.52 \mathrm{~mm}^{3}$ and post 209.60 $\pm 20.58 \mathrm{~mm}^{3}$ ) (Figure 5). However, the mean ACV measurement increased, but not significantly $(P=0.217)$, after $0.5 \%$ cyclopentolate (pre 197.5 $\pm 14.20 \mathrm{~mm}^{3}$ and post $201.1 \pm 13.27 \mathrm{~mm}^{3}$ ). The mean CCT measurement was decreased following $1 \%$ cyclopentolate (pre 553.93 $\pm 29.25 \mu$ and post 549.5 
Table I Parameter Change and p-value Between 0.5\% and I\% Cyclopentolate of the Hyperopic Group

\begin{tabular}{|c|c|c|c|c|c|c|c|c|}
\hline \multirow[b]{2}{*}{ Parameter } & \multicolumn{4}{|c|}{ Cyclopentolate I\% } & \multicolumn{4}{|c|}{ Cyclopentolate $0.5 \%$} \\
\hline & Pre & Post & $95 \% \mathrm{Cl}$ & $P$ & Pre & Post & $95 \% \mathrm{Cl}$ & $P$ \\
\hline Anterior chamber angle & $36.20 \pm 2.62$ & $34.82 \pm 2.85$ & $-0.63,3.38$ & 0.156 & $34.80 \pm 6.01$ & $33.90 \pm 7.17$ & $-1.21,2.99$ & 0.378 \\
\hline Anterior chamber depth* & $2.76 \pm 0.28$ & $2.89 \pm 0.25$ & $-0.18,-0.08$ & $0.000^{*}$ & $2.7 I \pm 0.28$ & $2.86 \pm 0.27$ & $-0.2 \mathrm{I},-0.08$ & 0.000 \\
\hline Anterior chamber volume & $|4| .40 \pm 20.6$ & $154.35 \pm 19.7$ & $-|8.85,-7.4|$ & 0.000 & $137.40 \pm 20.5$ & $152.93 \pm 20.5$ & $-20.6,-10.5$ & 0.000 \\
\hline Central corneal thickness & $568.93 \pm 31.2$ & $571.13 \pm 30.8$ & $-4.84,0.44$ & 0.096 & $568.13 \pm 34.0$ & $568.5 \pm 31.5$ & $-4.44,3.77$ & 0.864 \\
\hline Intraocular pressure & $13.25 \pm 1.77$ & $15.13 \pm 2.49$ & $-3.09,-0.67$ & 0.005 & $14.05 \pm 2.03$ & $|4.74 \pm| .84$ & $-1.36,0.01$ & 0.054 \\
\hline Refractive error & $+2.36 \pm 2.23$ & $+3.18 \pm 2.36$ & $-1.09,-0.54$ & $<0.001$ & $2.40 \pm 2.23$ & $3.20 \pm 2.76$ & $-1.27,-0.34$ & 0.002 \\
\hline Pupil diameter & $2.75 \pm 0.34$ & $4.42 \pm 1.23$ & $-2.33,-1.02$ & $<0.001$ & $2.78 \pm 0.35$ & $4.12 \pm 0.99$ & $-1.90,-0.78$ & $<0.001$ \\
\hline
\end{tabular}

Note: $* P$ values for differences in the anterior chamber depth before and after the administration of $1 \%$ cyclopentolate in the hypermetropic group.

$\pm 29.33 \mu$ ), and the mean difference was statistically significant, $P<0.001$. In contrast, the mean CCT measurement showed a slight increase after cyclopentolate $0.5 \%$ (pre $550.0 \pm 26.71 \mu$ and post $551.2 \pm 26.52 \mu$ ), and the mean difference was not statistically significant, $P=0.051$, as shown in Table 2 .

The mean IOP measurement of the myopic group showed a significant increase $(P<0.001)$ following $1 \%$ cyclopentolate (pre $14.74 \pm 2.51 \mathrm{mmHg}$ and post 15.97 $\pm 2.28 \mathrm{mmHg}$ ). However, the mean IOP measurement slightly decreased $(P=0.110)$ after $0.5 \%$ cyclopentolate (pre $13.92 \pm 1.38 \mathrm{mmHg}$ and post $13.55 \pm 1.66 \mathrm{mmHg}$ ). Regarding the mean change in the refractive error for both dosages of cyclopentolate, there was a significant decrease $(P<0.001)$ in myopia. The mean pupil size measurements of the myopic group significantly increased $(P<0.001)$ following both dosages of cyclopentolate, as shown in Table 2.

\section{Discussion}

Cyclopentolate agents have been clinically used to routinely assess ocular health. However, the impact of

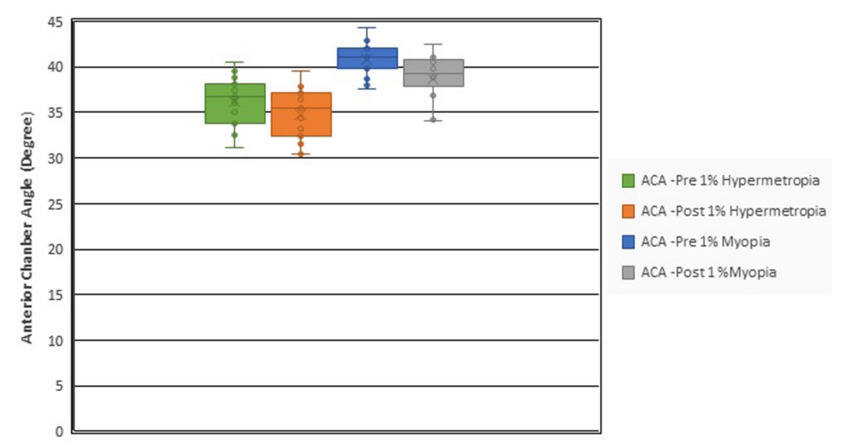

Figure I The anterior chamber angle (ACA) measurements (Y-axis) before and after the administration of $1 \%$ cyclopentolate, in both the myopic and hyperopic groups. It can be observed the slight differences in the measurements in each group before and after the administration of $1 \%$ cyclopentolate $(p>0.05)$. concentrations that are commonly used, $1 \%$ and $0.5 \%$, on anterior segment parameters and IOP relative to refractive error is still unclear. This study therefore attempted to provide a better understanding of the impact of cyclopentolate hydrochloride $1 \%$ and $0.5 \%$ on anterior chamber depth (ACD), anterior chamber volume (ACV), anterior chamber angle (ACA), central corneal thickness (CCT) and intraocular pressure (IOP). We found that, in myopic eyes, $0.5 \%$ cyclopentolate had less effect than $1 \%$ on ACV and IOP measurements, whereas little differences were found in the other parameters between the two concentrations. For the hypermetropic eyes, $0.5 \%$ had less effect than $1 \%$ only on the IOP measurements, whereas little differences were found in the other parameters between the two concentrations. However, overall, looking at some changes (pre-post values), they are mostly very small which does not look to have any clinical importance or impact.

The results of this study among the hypermetropic group showed significant changes in the measurements before and after the administration of $1 \%$ cyclopentolate

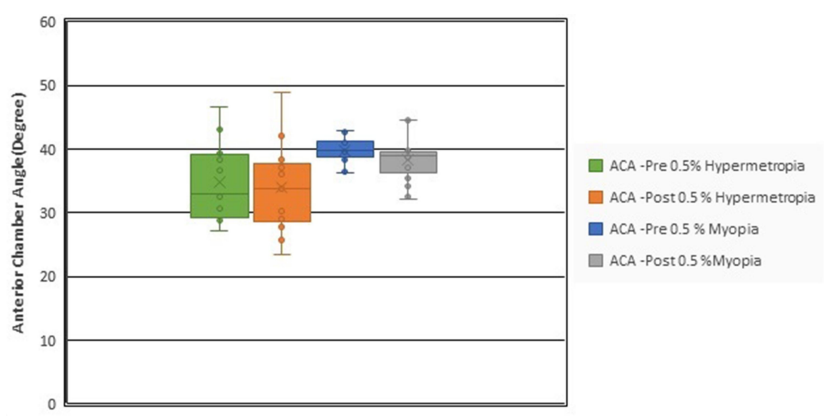

Figure 2 The anterior chamber angle (ACA) measurements ( $\mathrm{Y}$-axis) before and after the administration of $0.5 \%$ cyclopentolate, in both the myopic and hyperopic groups. It can be observed the slight differences in the measurements in each group before and after the administration of $0.5 \%$ cyclopentolate $(p>0.05)$. 


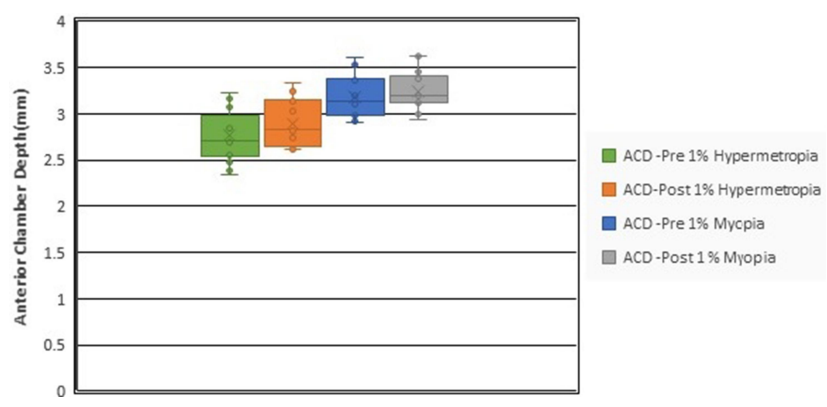

Figure 3 The anterior chamber depth (ACD) measurements (Y-axis) before and after the administration of $1 \%$ cyclopentolate, in both the myopic and hyperopic groups. There were significant differences in the measurements in each group before and after the administration of $1 \%$ cyclopentolate $(p<0.05)$.

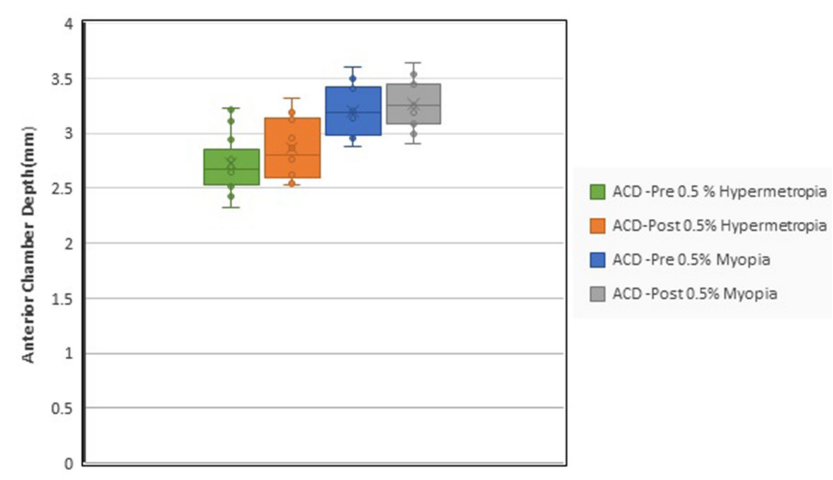

Figure 4 The anterior chamber depth ( $A C D$ ) measurements (Y-axis) before and after the administration of $0.5 \%$ cyclopentolate, in both the myopic and hyperopic groups. There were significant differences in the measurements in each group before and after the administration of $0.5 \%$ cyclopentolate $(p<0.05)$.

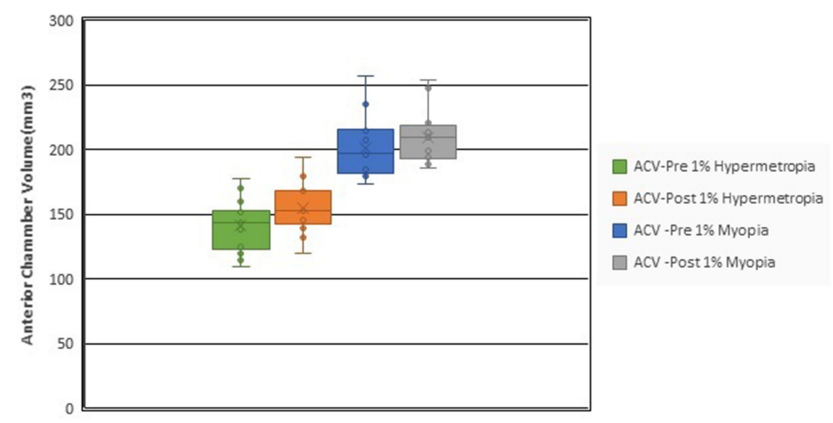

Figure 5 The anterior chamber volume (ACV) measurements, represented on the Y-axis, before and after the administration of I\% cyclopentolate, in both the myopic and hyperopic groups. There were significant differences in the measurements in each group before and after the administration of $1 \%$ cyclopentolate $(p<0.05)$.

in all anterior segment parameters, except ACA and central corneal thickness measurements (Table 1). On the other hand, there were significant changes in the measurements before and after the administration of $0.5 \%$ cyclopentolate in all anterior segment parameters, except ACA, $\mathrm{CCT}$ and IOP measurements. In the myopic group, there

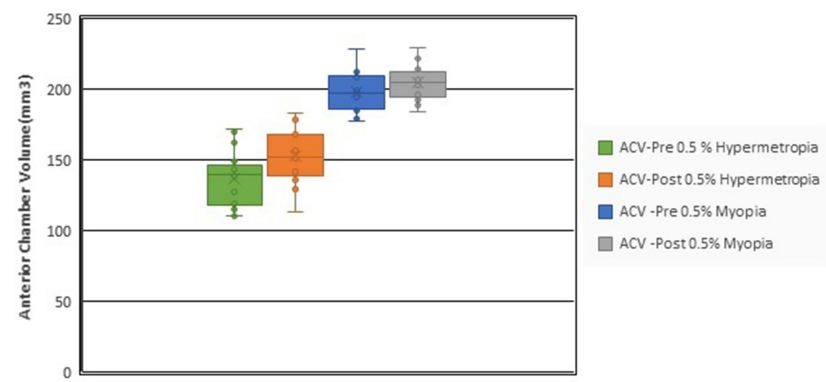

Figure 6 The anterior chamber volume (ACV) measurements, represented on the Y-axis, before and after the administration of $0.5 \%$ cyclopentolate, in both the myopic and hyperopic groups. There were significant differences in the measurements before and after the administration of $0.5 \%$ cyclopentolate in the hypermetropic group $(p<0.05)$, while there was no significant difference in the myopic group $(p>0.05)$.

were significant changes in the measurements before and after the administration of $1 \%$ cyclopentolate for all anterior segment parameters (Table 2). For $0.5 \%$ cyclopentolate, there were significant changes before and after the administration of the drop in all parameters, except ACV, CCT and IOP. These results indicate that both concentrations may make significant changes before and after measurements of the anterior segment parameters. However, $0.5 \%$ cyclopentolate may be preferred for use in order to reduce the effect of the cycloplegia on the IOP measurement in the myopic group.

In this study, we found that, in the hypermetropia and myopia groups, ACA had the only reduction in the measurements among the anterior segment parameters after we administered $1 \%$ cyclopentolate (Figures 1 and 2). This is consistent with a prior study that showed a significant decrease in the ACA after the administration of $1 \%$ cyclopentolate. ${ }^{2,19,20}$ Other studies showed a significant increase in the ACA measurements. ${ }^{7,221}$ However, these studies were conducted in children where there might have been anatomical changes and may explain the difference in the result that we found. For the rest of the anterior segment parameters, we found similar results found by the previously mentioned studies.

We noticed that there was a significant decrease in the CCT measurements after $1 \%$ instillations in the myopic group (Table 2). This is inconsistent with the results reported by several studies where they found an increase in the CCT after the administration of $1 \%$ cyclopentolate. ${ }^{2,9}$ The authors attribute this difference in CCT to the fact the previous studies were conducted in healthy young children where there could have been anatomical changes and may explain the difference in the result. However, the current study was done in myopic 
Table 2 Parameter Change and p-value Between 0.5\% and I\% Cyclopentolate of the Myopic Group

\begin{tabular}{|c|c|c|c|c|c|c|c|c|}
\hline \multirow[b]{2}{*}{ Parameter } & \multicolumn{4}{|c|}{ Cyclopentolate $1 \%$} & \multicolumn{4}{|c|}{ Cyclopentolate $0.5 \%$} \\
\hline & Pre & Post & $95 \% \mathrm{Cl}$ & $P$ & Pre & Post & $95 \% \mathrm{Cl}$ & $P$ \\
\hline Anterior chamber angle & $40.82 \pm 1.90$ & $38.80 \pm 2.65$ & $\mathrm{I} .04,3.0 \mathrm{I}$ & 0.001 & $39.78 \pm 2.08$ & $38.25 \pm 3.60$ & $0.32,2.73$ & 0.170 \\
\hline Anterior chamber depth* & $3.18 \pm 0.22$ & $3.25 \pm 0.21$ & $-0.09,-0.03$ & $0.001 *$ & $3.200 \pm 0.22$ & $3.26 \pm 0.05$ & $-0.09,-0.22$ & 0.003 \\
\hline Anterior chamber volume & $201.60 \pm 23.5$ & $209.60 \pm 20.6$ & $-11.83,-4.16$ & 0.001 & $197.5 \pm 14.2$ & $201.1 \pm 13.3$ & $-|0.2,-3.3|$ & 0.217 \\
\hline Central corneal thickness & $553.93 \pm 29.3$ & $549.5 \pm 29.33$ & $2.22,6.58$ & 0.001 & $550.0 \pm 26.7$ & $551.2 \pm 26.5$ & $-2.40,0.01$ & 0.051 \\
\hline Intraocular pressure & $|4.74 \pm 2.5|$ & $15.97 \pm 2.28$ & $-1.76,-0.69$ & $<0.001$ & $13.92 \pm 1.38$ & $13.55 \pm 1.66$ & $-0.09,0.84$ & 0.110 \\
\hline Refractive error & $-2.13 \pm 1.13$ & $-1.73 \pm 1.12$ & $-0.02,0.49$ & $<0.001$ & $1.99 \pm 1.26$ & $1.67 \pm 1.18$ & $0.22,0.42$ & $<0.001$ \\
\hline Pupil diameter & $3.05 \pm 0.63$ & $4.39 \pm 0.75$ & $-1.60,-1.07$ & $<0.001$ & $3.21 \pm 0.58$ & $4.25 \pm 0.79$ & $-138,-0.70$ & $<0.001$ \\
\hline
\end{tabular}

Note: $* P$ values for differences in the anterior chamber depth before and after the administration of $1 \%$ cyclopentolate in the myopic group.

adults. This issue is crucial because a change in the CCT affects the IOP measurements. Thick CCT is not a risk factor for increasing IOP, but thick CCT causes falsely high IOP measurement. In this study, we observed an increment in the IOP after the administration of $1 \%$ and $0.5 \%$ cyclopentolate in both the hyperopic and myopic groups. This is consistent with the previous reports that demonstrated an increment in the IOP with the use of $1 \%$ cyclopentolate. ${ }^{17,22}$ However, the application of $0.5 \%$ cyclopentolate did not cause a significant increment in the IOP, in both the hypermetropic and myopic groups.

Another effect of cyclopentolate drops may include changes in the refractive error. It can be observed that there were significant changes in the current study after the administration of $1 \%$ and $0.5 \%$ cyclopentolate in both the hypermetropic and myopic groups (Tables 1 and 2). Other studies ${ }^{8,20}$ reported a significant change in corneal curvature after installation of cycloplegia. The change in corneal curvature is due to paralysis of the ciliary muscle following cycloplegia, therefore, reducing its force on the sclera spur and resulting in corneal flattening. ${ }^{8}$ Although the change in refractive error could be a result of an alteration in the corneal curvature, it is more likely a result of the change in the lens position. The cycloplegic drug is known to induce backward lens iris diaphragm movement, and thus, subsequently increase ACD. Morton et $\mathrm{al}^{23}$ recommended the use of $0.5 \%$ cyclopentolate in young myopic patients, which led to accurate determination of refractive error without the effect of an accommodation system. In this study, we found a significant reduction in myopia after the administration of $0.5 \%$ cyclopentolate.

This study has several limitations. First, the sample size was small, so the results of this study are not robust to guide clinicians on which concentration should be used. Further studies with larger sample size are warranted in order to potentially guide clinicians to the better use of cyclopentolate. Another limitation is related to the fact that this study was only conducted among male participants. Future studies may be needed to assess if there would an effect of gender on the measurement after the administration of cyclopentolate. ACA and IOP in this study were not measured by the standard methods. Moreover, the current study did not categorize the degree of the refractive error and the size of eyes as referred to the axial length, for example. It might be worthwhile for the future study to look at the difference between mild, moderate and severe refractive errors, in terms of the effect of cyclopentolate. It would be of interest to compare other cycloplegic agents to cyclopentolate in order to strengthen the knowledge regarding the clinical use of cyclopentolate.

\section{Conclusion}

A topical dosage of $1 \%$ cyclopentolate showed significant changes in most parameters of the anterior segment in addition to the IOP among the myopic and hypermetropic groups. We found that $0.5 \%$ cyclopentolate may have less effect on the anterior segment parameters and IOP measurements. Further research is warranted in order to potentially investigate the clinical use of $0.5 \%$ cyclopentolate, as compared to $1 \%$ cyclopentolate and other cycloplegic agents. This can be accomplished by analyzing the effect of the $0.5 \%$ cyclopentolate on the anterior segment parameters and IOP measurements.

\section{Acknowledgments}

We are grateful to the staff and students of the Department of Optometry, Qassim University who helped us in the process of data collection. We extend our sincere thanks to 
Abdulmohsen Mubarak Alharbi and Abdulelah Abdulrahman Alshumaym for their help in data collection. We would also like to thank all the students and teachers who participated in this study.

\section{Author Contributions}

Waleed M. Alghamdi: Conception of study, study design, data collection, approval of final manuscript; Saif $\mathrm{H}$ Alrasheed: Conception of study, study design, data analysis, drafting of manuscript; Vishakh Nair: Conception of study, study design, data collection, drafting of manuscript; Muhammed S. Alluwimi: Conception of study, data analysis, editing of manuscript. All authors made substantial contributions to conception and design, acquisition of data, or analysis and interpretation of data; took part in drafting the article or revising it critically for important intellectual content; agreed to submit to the current journal; gave final approval of the version to be published; and agree to be accountable for all aspects of the work.

\section{Disclosure}

The authors report no conflicts of interest for this work and declare that they have no financial or personal relationships that may have inappropriately influenced them in writing this article.

\section{References}

1. Ozcaliskan S, Yenerel NM. The effect of cycloplegia on biometric measurements using swept-source optical coherence tomographybased biometry. Clin Exp Optom. 2019;102(5):501-505. doi:10.1111/ cxo. 12888

2. Arici C, Turk A, Ceylan OM, Kola M, Hurmeric V. Effects of $1 \%$ cyclopentolate hydrochloride on anterior segment parameters obtained with pentacam in young adults. Arq Bras Oftalmol. 2014;77(4):228232. doi:10.5935/0004-2749.20140059

3. Ambrósio R, Valbon BF, Faria-Correia F, Ramos I, Luz A. Scheimpflug imaging for laser refractive surgery. Curr Opin Ophthalmol. 2013;24(4):310-320. doi:10.1097/ICU.0b013e3 283622a94

4. Zhao Q, Li NY, Zhong XW. Determination of anterior segment changes with pentacam after phacoemulsification in eyes with primary angle-closure glaucoma. Clin Exp Ophthalmol. 2012;40(8):786-791. doi:10.1111/j.1442-9071.2012.02806.x

5. Konstantopoulos A, Hossain P, Anderson DF. Recent advances in ophthalmic anterior segment imaging: a new era for ophthalmic diagnosis? $\quad B r \quad J$ Ophthalmol. 2007;91(4):551-557. doi:10.1136/ bjo.2006.103408

6. Momeni-Moghaddam H, Maddah N, Wolffsohn JS, et al. The effect of cycloplegia on the ocular biometric and anterior segment parameters: a cross-sectional study. Ophthalmol Ther. 2019;8(3):387-395. doi:10.1007/s40123-019-0187-5
7. Niyaz L, Can E, Seymen Z, Eraydin B. Comparison of anterior segment parameters obtained by dual-scheimpflug analyzer before and after cycloplegia in children. J Pediatr Ophthalmol Strabismus. 2016;53(4):234-237. doi:10.3928/01913913-20160427-02

8. Cheng HC, Hsieh YT. Short-term refractive change and ocular parameter changes after cycloplegia. Optom Vis Sci. 2014;91(9):11131117. doi:10.1097/OPX.0000000000000339

9. Mutti DO, Zadnik K, Egashira S, Kish L, Twelker JD, Adams AJ. The effect of cycloplegia on measurement of the ocular components. Invest Ophthalmol Vis Sci. 1994;35(2):515-527.

10. Mohan K, Sharma A. Optimal dosage of cyclopentolate $1 \%$ for cycloplegic refraction in hypermetropes with brown irides. Indian $J$ Ophthalmol. 2011;59(6):514-516. doi:10.4103/0301-4738.86329

11. Ebri A, Kuper H, Wedner S. Cost-effectiveness of cycloplegic agents: results of a randomized controlled trial in Nigerian children. Invest Ophthalmol Vis Sci. 2007;48(3):1025-1031. doi:10.1167/iovs.060604

12. Palamar M, Egrilmez S, Uretmen O, Yagci A, Kose S. Influences of cyclopentolate hydrochloride on anterior segment parameters with pentacam in children. Acta Ophthalmol. 2011;89(5):e461-465. doi:10.1111/j.1755-3768.2011.02122.x

13. Arici C, Turk A, Ceylan OM, Kola M. The effect of topical $1 \%$ cyclopentolate on IOLMaster biometry. Optom Vis Sci. 2014;91 (11):1343-1347. doi:10.1097/OPX.0000000000000388

14. Özyol P, Özyol E, Baldemir E. Changes in ocular parameters and intraocular lens powers in aging cycloplegic eyes. Am J Ophthalmol. 2017;173:76-83. doi:10.1016/j.ajo.2016.09.032

15. Ceylan OM, Turk A, Erdurman C, et al. Comparison of oculus pentacam and stratus optical coherence tomography for measurement of central corneal thickness. Cornea. 2011;30(6):670-674. doi:10.1097/ICO.0b013e31820128a4

16. Rosenfield M, Linfield PBA. Comparison of the effects of cycloplegics on accommodation ability for distance vision and on the apparent near point. ophthalmic. Physiol Opt. 1986;6(3):317-320. doi:10.1111/j.1475-1313.1986.tb00722.x

17. Velasco Cabrera J, Eiroa Mozos P, Garcia Sanchez J, Bermudez Rodriguez F. Changes in intraocular pressure due to cycloplegia. Clao J. 1998;24(2):111-114.

18. Rosman MS, Skaat A, Chien JL, et al. Effect of cyclopentolate on in vivo Schlemm canal microarchitecture in healthy subjects. $J$ Glaucoma. 2017;26(2):133-137. doi:10.1097/IJG.000000000 0000599

19. Yuan Y, Zhang Z, Zhu J, et al. Responses of the ocular anterior segment and refraction to $0.5 \%$ tropicamide in chinese school-aged children of Myopia, Emmetropia, and Hyperopia. J Ophthalmol. 2015;2015:1-6.

20. Chang SW, Lo AY, Su PF. Anterior segment biometry changes with cycloplegia in myopic adults. Optom Vis Sci. 2016;93(1):12-18. doi:10.1097/OPX.0000000000000748

21. Güler E, Güragaç FB, Tenlik A, Yagci R, Arslanyilmaz Z, Balci M. Influences of topical cyclopentolate on anterior chamber parameters with a dual-scheimpflug analyzer in healthy children. $J$ Pediatr Ophthalmol Strabismus. 2015;52(1):26-30. doi:10.3928/0191391320141111-02

22. Oltulu R, Satirtav G, Kerimoglu H, Zengin N, Özkagnici A, Okka M. Influence of cyclopentolate hydrochloride on corneal biomechanical properties in healthy individuals. Eye Contact Lens. 2014;40(5):309311. doi:10.1097/ICL.0000000000000058

23. Morton M, Lee L, Morjaria P. Practical tips for managing myopia Community Eye Health. 2019;32(105):17-18. 


\section{Publish your work in this journal}

Clinical Ophthalmology is an international, peer-reviewed journal covering all subspecialties within ophthalmology. Key topics include: Optometry; Visual science; Pharmacology and drug therapy in eye diseases; Basic Sciences; Primary and Secondary eye care; Patient Safety and Quality of Care Improvements. This journal is indexed on PubMed
Central and CAS, and is the official journal of The Society of Clinical Ophthalmology (SCO). The manuscript management system is completely online and includes a very quick and fair peer-review system, which is all easy to use. Visit http://www.dovepress.com testimonials.php to read real quotes from published authors. 